\title{
Human Fetal Somatic and Visceral Morphometrics
}

\author{
MASON BARR, JR., WILL R. BLACKBURN, AND N. REEDE COOLEY, JR. \\ Departments of Pediatrics, Pathology, and Obstetrics, University of Michigan, Ann Arbor, Michigan 48109 (M.B.); \\ Department of Pathology, University of South Alabama, Mobile, Alabama 36617 (W.R.B., N.R.C.)
}

\begin{abstract}
Because of the increasing prenatal use of high-resolution ultrasonography to assess fetal growth and because growth aberration is one of the principal manifestations of abnormal intrauterine development, a more precise definition of normal growth is desirable. Our data set from autopsies of fetuses/neonates $(50-4,000 \mathrm{~g})$, collected with systematic attention to precision of measurement, is analyzed and presented. Previous computations of fetal growth curves appear to overstate the variation about the mean. Through the identification and exclusion of outliers and the use of appropriate curve fitting techniques, the problem of overestimation of variation about the mean has been largely eliminated. The new growth standards we present can be used to assess aberration from proportional growth and correlate it with gestational circumstances and particular morphological features of the fetus.

(c) 1994 Wiley-Liss, Inc.
\end{abstract}

There is a burgeoning interest in fetal examination, particularly to assess the accuracy of prenatal diagnosis, to evaluate the effects of prenatal management, and (at least from the parents' perspective) to find out "what went wrong" to cause a pregnancy loss. It is well known that one manifestation of abnormal gestational development is aberrant growth. With the increased use of high-resolution ultrasonography, the prospective study of human fetal growth has become an important topic of research, and detection of particular growth aberrations is increasingly proposed as an indication for more invasive prenatal diagnostics.

There is a considerable literature on intrauterine growth restriction, fetal macrosomia, and "symmetrical" vs. "asymmetrical" fetal growth. Most non-sonographic studies of somatic growth are based on a limited variety of measurements, typically body weight, crown-heel length, and head circumference. The majority of these studies have considered only those fetuses or newborns that fall into the "viable" age range. Several reports concern standards for visceral growth as measured at autopsy, again predominantly from fetuses/neonates in the viable range (Gruenwald and Minh, '60; Schulz et al., '62; Fujikura and Froelich, '72; Potter and Craig, '75; Larroche, '77; Singer et al., '91; Saul et al., '88; Hall et al., '89). A few reports deal with the very small or previable fetus only (Potter and Craig, '75; Singer et al., '91; Tanimura et al., '71; Golbus and Berry, '77; Burdi et al., '81; Shepard et al., '88). Because growth is not discontinuous across the limit of viability, particularly when that limit changes with the application of neonatal care technology, we believe there is a need for standards of growth, both somatic and visceral, that span the whole, or at least the postembryonic portion, of intrauterine development. One solution to this need would be to combine a set of standards derived from early fetuses with a set of standards derived from older fetuses/neonates. In attempting this with the available data, however, it is found that there are discontinuities between the mean expected measurements at the high end of one and the low end of the other. It is also apparent that the estimation of the variance about the mean varies so widely that no smooth juncture can be made. A problem with many published standards, particularly of visceral weights, is that the mathematical methods used inflate the estimate of variance about the mean. A commonly used method is to group a number of fetuses within a body weight range (e.g., 375 to $625 \mathrm{~g}$ ) and then to calculate the parameters of the observed organ weights for the midpoint weight $(500 \mathrm{~g})$ of the group (Gruenwald and Minh, '60; Schulz et al., '62; Fujikura and Froelich, '72; Potter and Craig, '75; Larroche, '77; Singer et al., '91). The practical effect of this method is to inflate the estimate of variance, not infrequently pushing the lower limit of the $95 \%$ prediction interval below zero ("Black Hole Phenomenon").

Several papers have reported fetal growth curves developed from data sets that were not fractionated into discontinuous weight categories. Most often these have used polynomial expressions to generate the curve (Tanimura et al., '71; Golbus and Berry, '77; Burdi et al., '81; Shepard et al., '88). A problem sometimes encountered with the polynomial method is that the variance may be underestimated at the low end and wildly

Received November 2, 1993; accepted January 31, 1994

Address reprint requests to Dr. Mason Barr, Jr., Teratology UnitPediatric Genetics, D1109 MPB Box 0718, University of Michigan, Ann Arbor, MI 48109. 
overestimated at the high end, even to the point that with increasing body weight "normal" organ weights decrease ("Wild Blue Yonder Phenomenon"). Most of the papers reporting the polynomial growth curves deal only with the low end of the fetal weight range (i.e., under $1,000 \mathrm{~g}$ body weight) (Tanimura et al., '71; Golbus and Berry, '77; Burdi et al., '81; Shepard et al., '88).

On close consideration of some of the available standards, we suspect that criteria for data inclusion may have been more relaxed than one would wish, so that clearly abnormal specimens were included in the data set. It is also possible or even likely that measurements collected by a number of different observers were not always determined by the same method or with the same assiduity.

We have sensed over the years that particular growth aberrations are associated with particular syndromes and gestational circumstances. We thought that a more precise quantification of the aberration would be useful in the study of intrauterine development. To do this, we required a more accurate definition of "normal" than is presently available. Accordingly we have surveyed our collections of fetal/neonatal autopsies for data from "normal" specimens in order to generate standards by which to assess growth.

\section{DATA COLLECTION}

The data were drawn from fetal/neonatal autopsies performed personally by the authors at the University of Michigan ( $\mathrm{n}=2,633$ ) and the University of South Alabama ( $\mathrm{n}=1,644)$. We will hereafter refer to fetuses and by that term mean to include neonates. All measurements were made by one of the three authors, using the same methods, and essentially the same equipment. For measurements that were collected by differing techniques, the data presented here are from only one center (arm length, Michigan). Weights were determined on volumetric (for weights over $100 \mathrm{~g}$ ) or electronic (for weights under $100 \mathrm{~g}$ ) balances. For these analyses, weights were measured to the milligram or to the third significant digit, whichever was larger, and recorded in grams. Length and circumference measurements were made using standard metric rulers, tapes and calipers and recorded in millimeters. No systematic assessment of measurement error was attempted, although spot checks indicated a $1-2 \%$ variation on repeat measurement by the same observer. To check for inter-observer difference in technique, we examined the data from the two centers for homogeneity and no apparent differences were found in the data reported here.

Specifics on measurement technique and sample preparation are as follows. Before the body was weighed, excess umbilical cord (to $1 \mathrm{~cm}$ ), cord clamps, tubes, catheters, tape, identification bands, and similar extraneous items were removed (or weighed separately later and subtracted). The crown-heel length was measured with the body fully extended in the supine position, from a vertical line touching the vertex to the sole of the foot held in the plantigrade position; a length measuring jig was used to eliminate parallax errors. The crown-rump length was measured in the same jig, from the vertex to a vertical line touching the perineum (scrotum retracted forward in males). The leg length is the difference between the crown-heel and crown-rump lengths, or from the perineum to the sole of the foot held in the plantigrade position. The arm length is from the angle of the arm-shoulder to the tip of the third digit, with the arm fully extended and parallel to the long axis of the body. The foot length is from the posterior aspect of the heel to the tip of the first toe.

The brain weight included all parts to the cervicomedullary junction but not any major intraventricular blood or fluid collection. The spleen was trimmed of extraneous hilar tissue but accessory splenules were included. The thymus was trimmed of surrounding adipose tissue. The thyroid was trimmed of overlying cervical muscle; parathyroids may be included. For the heart, the neck and arm vessels were transected to a length equivalent to the bifurcation of the innominate artery, the aorta a few millimeters distal to the ductus arteriosus, the superior vena cava at its confluence with the innominate vein, the inferior vena cava at the level of the diaphragm, and the pulmonary vessels midway between the heart and the hila of the lungs. Prior to weighing, the heart was opened and all contained blood was removed. The liver was left with $1-2 \mathrm{~cm}$ of umbilical vein attached and the gallbladder was not drained. The liver was allowed to drain passively through the superior vena cava by letting it stand inverted. The adrenals and kidneys were each trimmed of surrounding adipose tissue and a length of ureter to the lower renal pole was left attached. The renal vessels were transected near the hilum. The lungs included a short length of mainstem bronchus and pulmonary vessels; they were allowed to drain passively by placing them hilum down. As the fetal dissection proceeded, the various organs were placed on a moistened sponge to drain passively of contained blood while diminishing evaporative weight loss. Just prior to weighing, extraneous surface moisture and blood were blotted off on paper toweling.

\section{SPECIMEN SELECTION/EXCLUSION}

For initial consideration, only those nonmacerated, nonhydropic specimens that were morphologically normal or which had only one or two minor malformations (e.g., type-B postaxial polydactyly, telecanthus, common origin of the carotid arteries) were selected. Fetuses with abnormal karyotypes were excluded. Any infant, regardless of weight, who survived for more than 3 days was excluded from our study. For certain liveborns, data exclusions were made for selected or- 

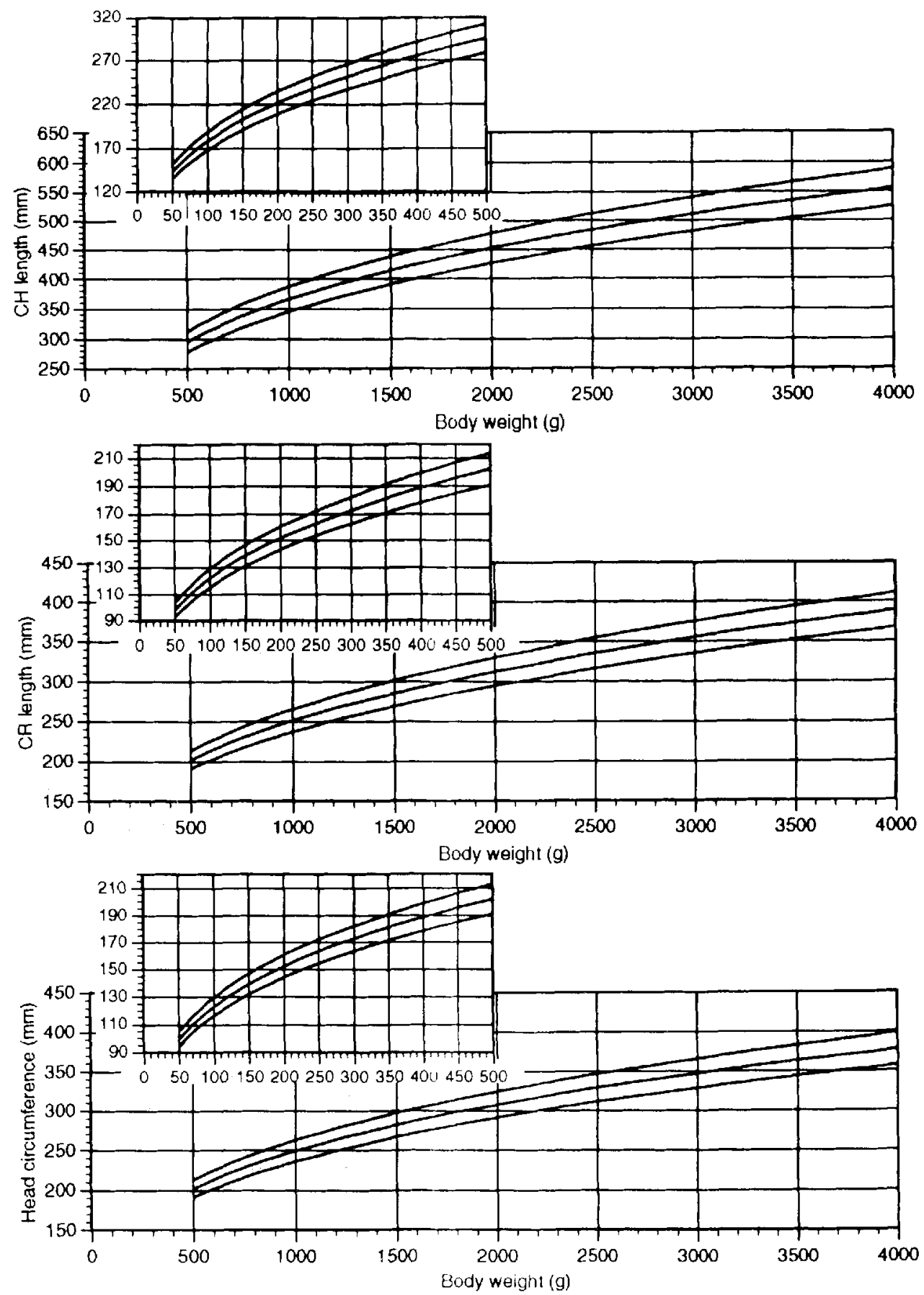

Fig. 1. Predicted means and $95 \%$ prediction intervals of crown-rump length, crown-heel length, and head circumference for body weight.

gans (e.g., lungs for massive pulmonary hemorrhage; see below).

The maternal histories were then reviewed, and fetuses from mothers having diabetes, chronic hypertension, or other disorders previously associated with abnormal fetal growth, (e.g., systemic lupus erythema- tosus) were excluded. Polyhydramnios, oligohydramnios (unless acute), and fetal growth restriction confirmed by serial ultrasonography were also bases for exclusion. The presence of acute oligohydramnios (less than 3 days), acute chorioamnionitis, acute maternal hypertension, and several other very recent 

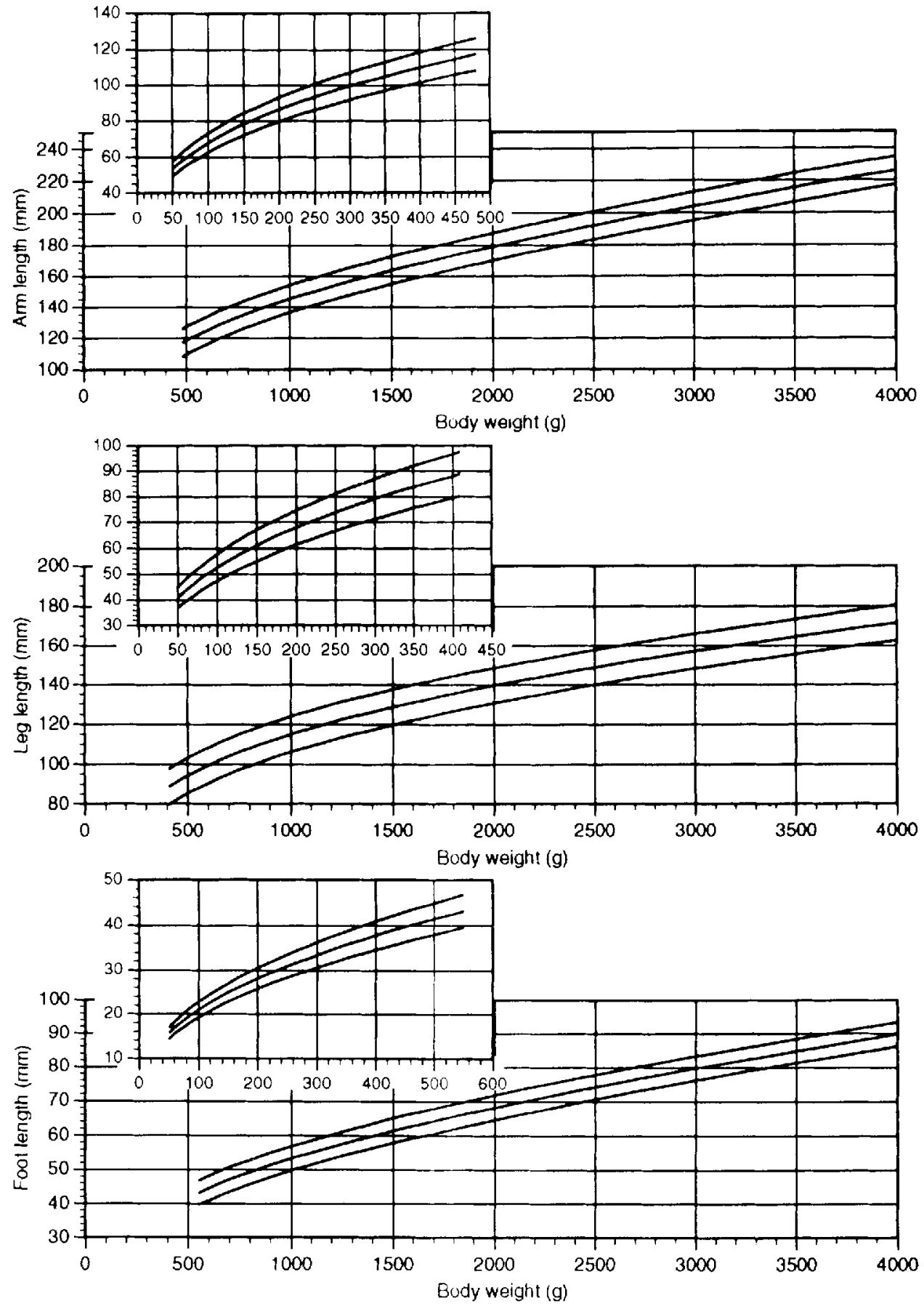

Fig. 2. Predicted means and $95 \%$ prediction intervals of arm length, leg length, and foot length for body weight.

onset maternal conditions were judged likely to have minimal impact on fetal growth and thus were not bases for exclusion; this assumption appears to be valid in that retrospective analysis shows no differences between such specimens and the rest of the sample.
The third step in sample selection was to make scatterplots of the data to identify grossly evident outliers (retrospectively, those data points that were greater than about five standard deviations from the mean). If a given fetus had only one such aberrant value, that particular measurement was excluded. If two or more 

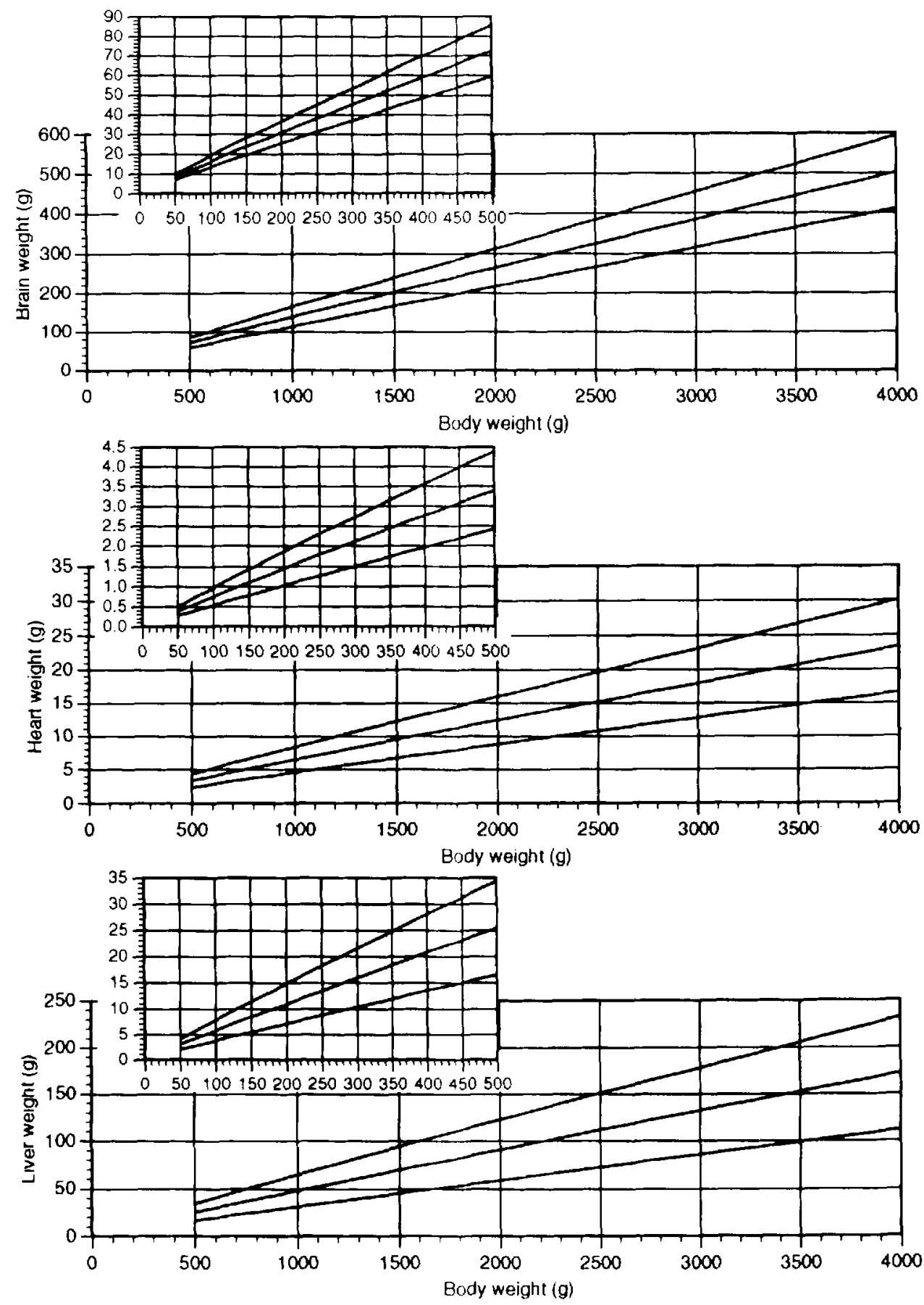

Fig. 3. Predicted means and $95 \%$ prediction intervals for weights of brain, heart, and liver for body weight.

aberrant values were present, the fetus was excluded from the study.

The fourth and final step in sample selection was performed during the calculation of the growth curves. Rough curve fitting was performed on the sample selected so far, and the data set was inspected for stan- dard scores outside the $98 \%$ prediction intervals. If only one or two measurements in a given fetus were aberrant, those measurements were excluded. If three or more measurements were aberrant, the fetus was dropped from the sample. After the above exclusions, 1,014 normal fetuses (671 Michigan, 343 Alabama, 

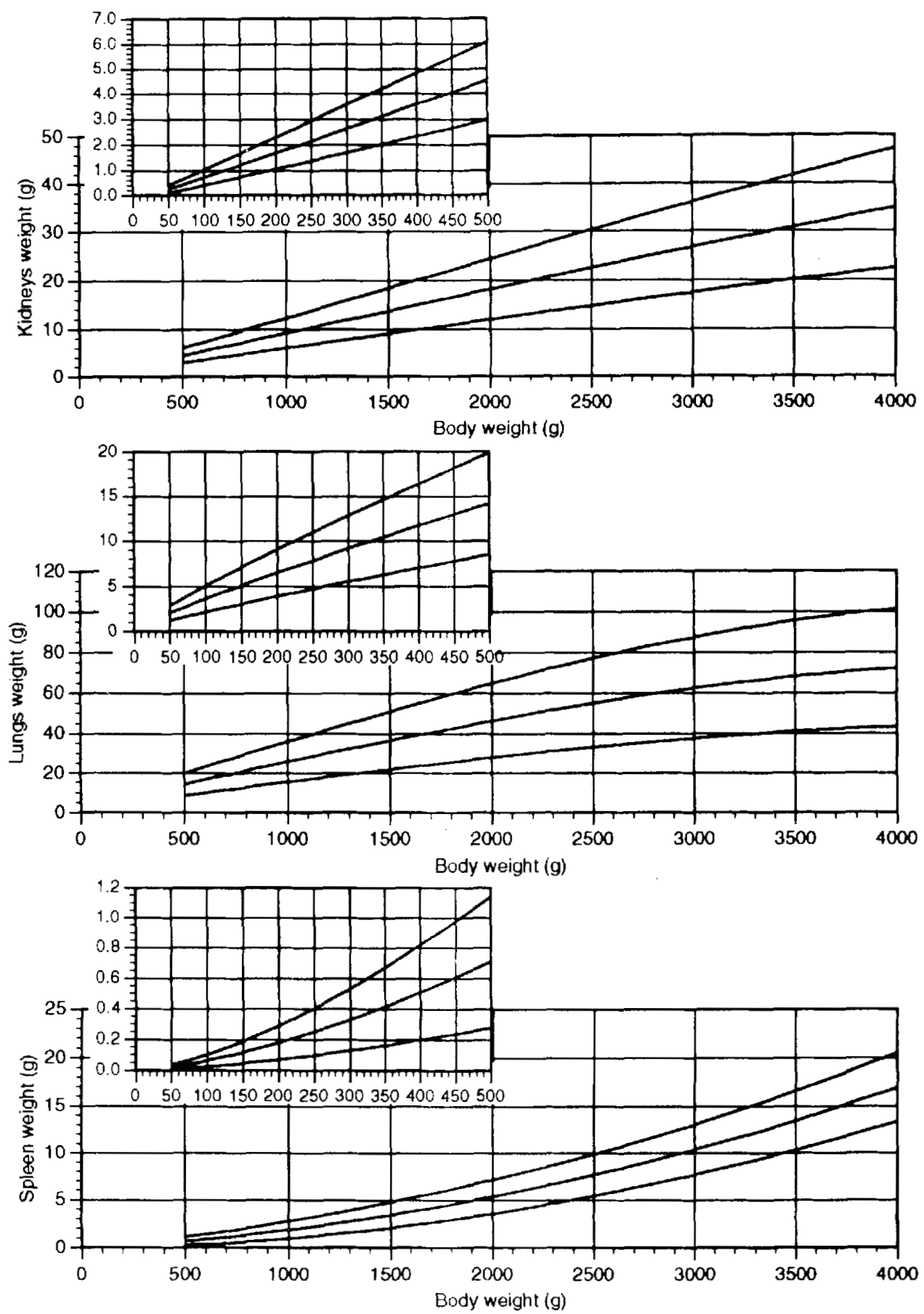

Fig. 4. Predicted means and 95\% prediction intervals for weights of kidneys (combined), lungs (combined), and spleen for body weight.

weight range $50-4,000 \mathrm{~g}$ ) remained to establish normal standards for body measurements and visceral weights. The sex distribution was, Michigan: 366 male, 305 female; Alabama: 192 male, 151 female. The race distribution was, Michigan: 468 White, 188 Black, 15 other; Alabama: 110 White, 229 Black, 4 other.

\section{CURVE CALCULATION}

In the past, mathematical models were viewed as representing the principles or laws of growth. More recently, they have come to be seen as tools for biologic analysis (Zeger and Harlow, '91). This means that the 

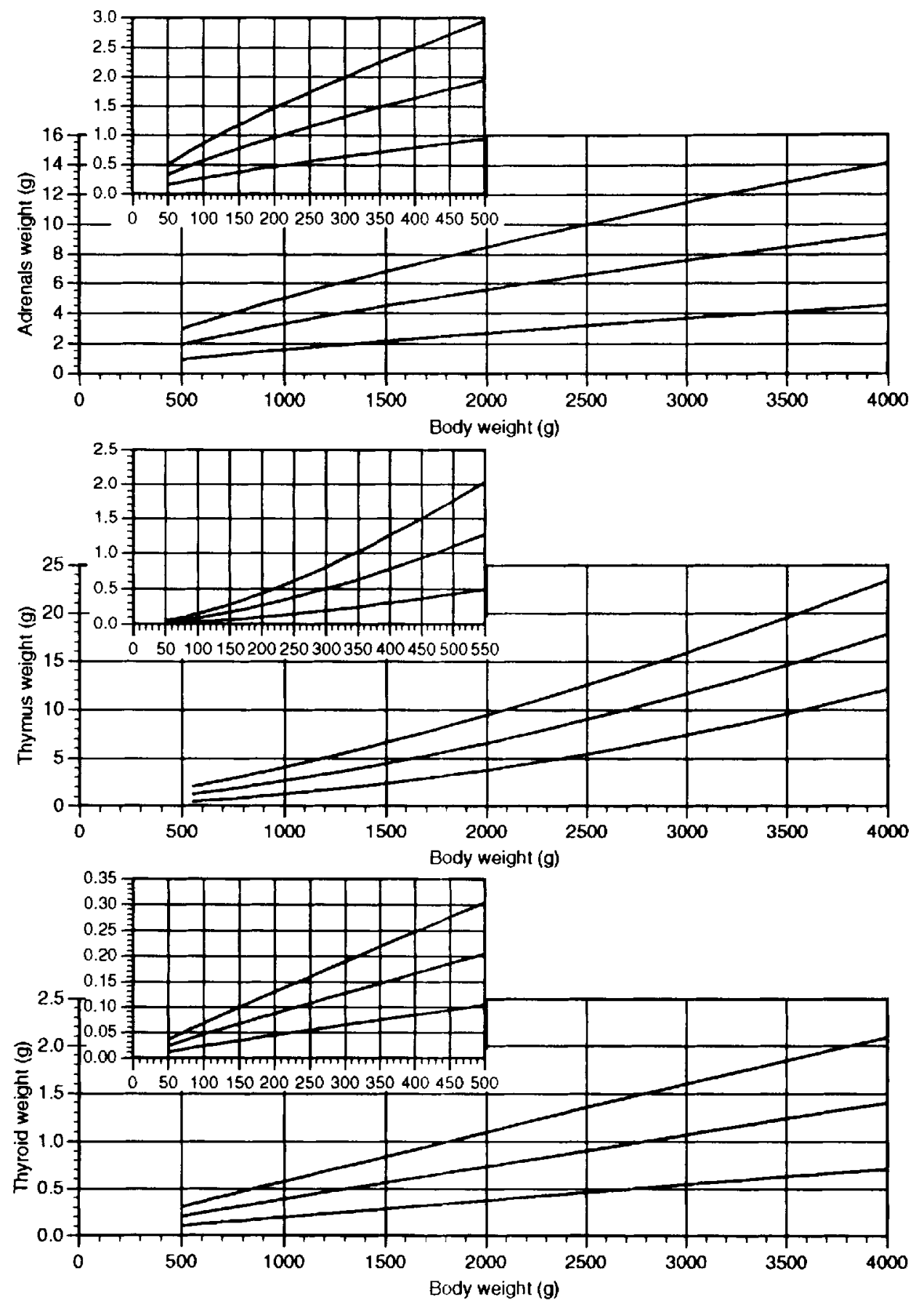

Fig. 5. Predicted means and 95\% prediction intervals for weights of adrenals (combined), thymus, and thyroid for body weight.

mathematics of curve fitting do not have to conform to some preconceived notion of how growth should be, but are free to be used to describe what was actually observed. 'To avoid the problems associated with previously published standards, we sought a method that would provide a tight fit to the data and be applicable over a wide range of fetal sizes. Certain basic assumptions were made. It was assumed that measurements made of each fetus at a single point in time would in aggregate be a true reflection of what those measurements would have been were it possible to determine them serially in the same fetuses. It was assumed that 
TABLE 1. Formulas and constants for somatic measurements (in millimeters or grams) ${ }^{1}$

\begin{tabular}{|c|c|c|c|c|c|c|}
\hline $\mathrm{Y}$ & Formula $^{2}$ & a & b & c & d & $\mathrm{e}$ \\
\hline \multicolumn{7}{|c|}{ When $\mathrm{X}=$ body weight } \\
\hline CHL & 2 & 42.13 & 0.3137 & $-6.57 \times 10^{-7}$ & - & 1.235 \\
\hline CRL & 2 & 28.91 & 0.313 & $9.8 \times 10^{-8}$ & 一 & 0.8317 \\
\hline OFC & 2 & 29.73 & 0.3086 & $-5.44 \times 10^{7}$ & - & 0.8159 \\
\hline ARM $(X<480)$ & $\overline{1}$ & 13.78 & 0.3465 & - & - & 0.5427 \\
\hline $\operatorname{ARM}(\mathrm{X}>480)$ & 6 & -66.56 & $1.374 \times 10^{-2}$ & 28.65 & - & 4.492 \\
\hline LEG $(X<410)$ & 3 & 10.32 & 0.3511 & $3.14 \times 10^{-5}$ & $-3.7 \times 10^{8}$ & 0.5165 \\
\hline LEG $(X>410)$ & 6 & -61.34 & $7.615 \times 10^{-3}$ & 24.43 & - & 4.539 \\
\hline FTL $(X<550)$ & 2 & 2.988 & 0.4236 & $-9.93 \times 10^{7}$ & - & 0.1275 \\
\hline FTL $(\mathrm{X}>550)$ & 6 & -36.01 & $6.65 \times 10^{-3}$ & 11.96 & - & 1.829 \\
\hline BRN & 1 & 0.2237 & 0.9304 & - & - & $2.072 \times 10^{2}$ \\
\hline \multicolumn{7}{|c|}{ When $\mathrm{X}=\mathrm{C}-\mathrm{R}$ length } \\
\hline BDY & 1 & $2.68 \times 10^{-5}$ & 3.153 & - & - & $2.455 \times 10^{-6}$ \\
\hline $\mathrm{CHL}$ & 3 & 1.374 & 0.9973 & $1.364 \times 10^{-4}$ & $-3.1 \times 10^{-7}$ & $2.341 \times 10^{-2}$ \\
\hline $\mathrm{OFC}$ & 3 & 1.112 & 0.9662 & $1.293 \times 10^{-4}$ & $-2.94 \times 10^{7}$ & $3.879 \times 10^{-2}$ \\
\hline ARM & 3 & 0.4584 & 1.017 & $2.777 \times 10^{-4}$ & $-6.49 \times 10^{-7}$ & $1.91 \times 10^{-2}$ \\
\hline LEG & 3 & 0.375 & 0.9929 & $4.385 \times 10^{1}$ & $-9.95 \times 10^{-7}$ & $2.056 \times 10^{-2}$ \\
\hline FTL & 3 & $4.602 \times 10^{-2}$ & 1.239 & $3.849 \times 10^{-4}$ & $-8.77 \times 10^{-7}$ & $2.271 \times 10^{3}$ \\
\hline BRN & 1 & $1.302 \times 10^{-5}$ & 2.926 & - & - & $1.343 \times 10^{-6}$ \\
\hline \multicolumn{7}{|c|}{ When $\mathrm{X}=$ brain weight } \\
\hline BDY & 1 & 5.54 & 1.051 & - & - & 0.5397 \\
\hline CHL & 3 & 70.9 & 0.3308 & $3.746 \times 10^{5}$ & $-1.10 \times 10^{-7}$ & 2.459 \\
\hline CRL & 1 & 48.42 & 0.3335 & - & - & 1.706 \\
\hline $\mathrm{OFC}$ & 3 & 49.72 & 0.3241 & $5.209 \times 10^{-5}$ & $-1.5 \times 10^{-7}$ & 1.504 \\
\hline ARM $(\mathrm{X}<63)$ & 3 & 24.87 & 0.3603 & $1.643 \times 10^{-4}$ & $-1.273 \times 10^{-6}$ & 1.046 \\
\hline $\operatorname{ARM}(\mathrm{X}>63)$ & 6 & -27.76 & $8.756 \times 10^{-2}$ & 32.6 & - & 4.71 \\
\hline LEG $(\mathrm{X}<61)$ & 3 & 18.82 & 0.3644 & $4.301 \times 10^{-4}$ & $-3.283 \times 10^{-6}$ & 0.9175 \\
\hline LEG $(X>61)$ & 6 & -27.45 & $4.982 \times 10^{2}$ & 27.51 & - & 4.422 \\
\hline FTL $(\mathrm{X}<85)$ & 3 & 6.04 & 0.4402 & $3.534 \times 10^{-4}$ & $-2.679 \times 10^{6}$ & 0.3138 \\
\hline FTL $(\mathrm{X}>85)$ & 6 & -26.2 & $3.597 \times 10^{-2}$ & 15.2 & - & 4.422 \\
\hline
\end{tabular}

${ }^{1} \mathrm{BDY}$, body weight; CHL, crown-heel length; CRL, crown-rump length; OFC, head circumference; ARM, arm length; LEG, leg length; FTL, foot length; BRN, brain weight.

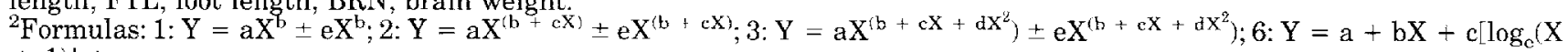
$+1)\rfloor \pm$ e.

fetal growth is effectively an orderly process without major intermittent accelerations or decelerations. It was assumed that, on average in any given fetus, the tendency would be for growth to follow a pattern such that, if a particular measurement were one standard deviation below the mean at an early stage of gestation, it would be one standard deviation below the mean at a later stage of gestation. It was assumed that the absolute variation about the mean would increase with fetal size/age and that the increase would have some mathematical relation to the size/age. In practice, it was empirically evident that certain measurements (arm, leg, foot, spleen, thymus) underwent a change, not so much in mean but in distribution about the mean, at a fetal size roughly equivalent to $500 \mathrm{~g}$ body weight. For the limb measurements, there was an initial expansion of the variance, followed by little or no change with increasing size/age beyond a certain point. For other measurements the assumption of continuous expansion of variance appeared to be correct, although for thymus and spleen the best mathematical description of that expansion changed. The actual shape of the growth curve was not assumed but was left to be discovered.
Curve fitting was done by testing unweighted and weighted polynomial equations and power equations with simple and polynomial exponents to achieve the best fit to the observed data, using the Multiple General Linear Hypothesis (Systat v5.1 for the Macintosh). A variety of formulas can be used to depict the mean with almost equal validity. Goodness of fit was assessed both visually and by analysis of variance of the regression. For the majority of analyses, power equations gave the best results. Because a major concern was with the variance about the mean, the power equation method used here was developed to reflect our assumption that, on average, the relative deviation from the mean would undergo no significant shift with increasing size/age. For this reason, in the power equations the shape of the curve is also built into the estimation of the variance, with the result that the mean of the residuals, when plotted against "X," is shown neither to increase nor decrease as " $\mathrm{X}$ " increases.

\section{FORMULAS TESTED}

$Y_{c}=a+b X+c X^{2}, \pm e$

$Y_{0}=a+b X+c X^{2}+d X^{3}, \pm e$ 
TABLE 2. Formulas and constants for visceral measurements (in grams) ${ }^{1}$

\begin{tabular}{|c|c|c|c|c|c|c|}
\hline $\mathrm{Y}$ & Formula ${ }^{2}$ & a & $\mathrm{b}$ & c & $\mathrm{d}$ & $\mathrm{e}$ \\
\hline \multicolumn{7}{|c|}{ When $\mathrm{X}=$ body weight } \\
\hline BRN & 1 & 0.2237 & 0.9304 & - & - & $2.072 \times 10^{2}$ \\
\hline SPL $(X<500)$ & 1 & $6.467 \times 10^{-5}$ & 1.4973 & - & - & $2.008 \times 10^{-5}$ \\
\hline SPL $(X>500)$ & 4 & $-6.802 \times 10^{-2}$ & $1.186 \times 10^{-3}$ & $7.67 \times 10^{-7}$ & - & $4.567 \times 10^{4}$ \\
\hline TYM $(\mathrm{X}<550)$ & 1 & $8.496 \times 10^{-5}$ & 1.523 & - & - & $2.637 \times 10^{-5}$ \\
\hline TYM $(\mathrm{X}>550)$ & 4 & 0.1726 & $2.31 \times 10^{-3}$ & $5.45 \times 10^{7}$ & $\ldots$ & $7.228 \times 10^{4}$ \\
\hline TYR & 2 & $6.348 \times 10^{-4}$ & 0.9292 & $-1.49 \times 10^{-7}$ & - & $1.588 \times 10^{-4}$ \\
\hline HRT & 2 & $1.01 \times 10^{-2}$ & 0.937 & $-7.43 \times 10^{7}$ & - & $1.493 \times 10^{3}$ \\
\hline LIV & 2 & $8.469 \times 10^{-2}$ & 0.9184 & $3.9 \times 10^{-8}$ & - & $1.511 \times 10^{-2}$ \\
\hline ADR & 2 & $1.642 \times 10^{-2}$ & 0.7691 & $-1.106 \times 10^{-6}$ & - & $4.317 \times 10^{-3}$ \\
\hline KID & 4 & -0.2429 & $9.642 \times 10^{-3}$ & $-2.13 \times 10^{7}$ & - & $1.585 \times 10^{-3}$ \\
\hline LNG & 3 & $7.668 \times 10^{-2}$ & 0.8359 & $9.477 \times 10^{-6}$ & $-3.0 \times 10^{-9}$ & $1.559 \times 10^{-2}$ \\
\hline \multicolumn{7}{|c|}{ When $\mathrm{X}=$ brain weight } \\
\hline BDY & 1 & 5.5396 & 1.051 & - & - & 0.5397 \\
\hline SPL $(X<100)$ & 1 & $8.629 \times 10^{-4}$ & 1.567 & - & - & $2.754 \times 10^{-4}$ \\
\hline SPL $(X>100)$ & 5 & $-3.04 \times 10^{-2}$ & $4.878 \times 10^{-3}$ & $7.933 \times 10^{-5}$ & $-7.8 \times 10^{-8}$ & $3.183 \times 10^{-3}$ \\
\hline TYM $(X<76)$ & 1 & $1.106 \times 10^{-3}$ & 1.617 & - & - & $3.853 \times 10^{-4}$ \\
\hline TYM $(\mathrm{X}>76)$ & 4 & -0.1934 & $1.568 \times 10^{-2}$ & $3.451 \times 10^{-5}$ & - & $5.419 \times 10^{-3}$ \\
\hline TYR & 1 & $2.961 \times 10^{-3}$ & 0.9894 & - & - & $7.713 \times 10^{11}$ \\
\hline HRT & 3 & $4.688 \times 10^{-2}$ & 0.9998 & $3.082 \times 10^{-5}$ & $-9.3 \times 10^{-8}$ & $7.136 \times 10^{-3}$ \\
\hline LIV & 2 & 0.3877 & 0.9783 & $4.628 \times 10^{-6}$ & - & $7.097 \times 10^{-2}$ \\
\hline ADR & 3 & $5.959 \times 10^{-2}$ & 0.8123 & $3.168 \times 10^{-5}$ & $-7.6 \times 10^{8}$ & $1.575 \times 10^{-2}$ \\
\hline KID & 4 & -0.3577 & $6.674 \times 10^{-2}$ & $6.873 \times 10^{-6}$ & - & $1.177 \times 10^{-2}$ \\
\hline LNG & 3 & 0.3155 & 0.8817 & $9.087 \times 10^{5}$ & $-2.21 \times 10^{7}$ & $6.652 \times 10^{-2}$ \\
\hline
\end{tabular}

${ }^{1}$ BDY, body weight; BRN, brain; SPL, spleen; TYM, thymus; TYR, thyroid; HRT, heart; LIV, liver; ADR, adrenals combined; KID, kidneys combined; LNG, lungs combined.

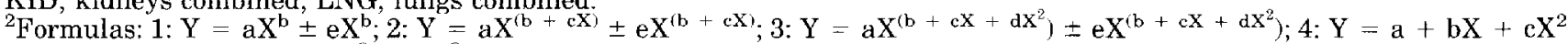
$\pm \mathrm{eX} ; 5: \mathrm{Y}=\mathrm{a}+\mathrm{bX}+\mathrm{cX}^{2}+\mathrm{dX}^{3} \pm \mathrm{eX}$.

$\mathrm{Y}_{\mathrm{e}}=\mathrm{aX}+\mathrm{bX}^{2}, \pm \mathrm{eX}$

$Y_{\mathrm{e}}=\mathrm{aX}+\mathrm{bX}^{2}+\mathrm{cX}^{3}, \pm \mathrm{eX}$

$Y_{e}=a X+b X^{2}+c X^{3}+d X^{4}, \pm e X$

$Y_{\mathrm{e}}=\mathrm{a} X^{\mathrm{b}}, \pm \mathrm{eX}^{\mathrm{b}}$

$\mathrm{Y}_{\mathrm{e}}=\mathrm{a} \mathrm{X}^{(\mathrm{b}+\mathrm{c} X)}, \pm \mathrm{eX}^{(\mathrm{b} \mid \mathrm{c} \mathrm{X})}$

$Y_{e}=a X^{\left(b+c x+d X^{2}\right)}, \pm e X^{\left(b+c x । d X^{2}\right)}$

$\mathrm{Y}_{\mathrm{e}}=\mathrm{a}+\mathrm{bX}+\mathrm{c}\left[\log _{\mathrm{e}}(\mathrm{X}+1)\right], \pm \mathrm{e}$

Where $Y_{e}$ is the expected value of the dependent variable, $X$ is the observed value of the independent variable (body weight, crown-rump length, or brain weight), and $a, b, c$, and $d$ are constants describing the curve and $\mathrm{e}$ is a constant describing the standard deviation.

\section{DISCUSSION}

Emphasis in these studies has been on the proportionality of fetal growth, irrespective of gestational age. Although we have a good amount of reliable age data, for many of the fetuses/infants we have examined there are obvious errors in the clinical statements of age. In this context, we have chosen to look at intrafetal proportionality and leave the correlation with actual gestational age for separate consideration. Accordingly, our analyses have used body weight, brain weight, and crown-rump length as primary standards against which to assess other measurements.

Many maternal factors influence fetal growth, such as pregravid weight, weight gain, diet, parity, race, smoking, medications, and uteroplacental blood flow.
Fetal factors, such as sex, also influence growth. These factors are not treated separately in our analysis, for our aim is not to define ideal fetal growth, but rather to give the perinatal pathologist/teratologist a closer approximation of normal than is currently available. $\mathrm{Ob}-$ taining a sufficient sample of truly normal human fetuses may not be possible. Virtually all of the fetuses we have examined have some gestational circumstances that raise questions about normality. It may be more accurate to describe our findings as "typical," but we sense from the accumulated data that these really were normal and have chosen to call them that. The standards we are reporting are restricted to fetuses weighing between 50 and $4,000 \mathrm{~g}$. Our sample is heavily weighted toward the lighter/smaller end. For obvious reasons, normal fetuses of more advanced gestational ages uncommonly come to autopsy. The situation is such that the majority of fetuses that we examined weighing more than $1,000 \mathrm{~g}$ were excluded using the above criteria. However, the curve generating methods that we have used compensate for this high-end sample deficiency through anchoring the curve and its estimated variance by the mass of data at the low and midrange.

We acknowledge that fetuses with "symmetrical" growth restriction may be included in our sample, but our experience is that what has been called symmetrical rarely is so when multiple variables are considered (Johnson et al., '89). These fetuses were examined for 
homogeneity of growth, as described above, and they were excluded from the analyses if more than one or two disproportionate measurements were found. Although we have not controlled for various maternal and fetal factors (race, fetal sex, and maternal smoking, weight, weight gain, and parity), the standards we propose can be used in the study of individual cases by comparing them with the observed morphometrics to help make inferences about the effects of such maternal and fetal factors.

We have elected to present only a small sample of our growth curves as graphs (Figs. 1-5). The calculation methodology used, although dauntingly complex as a paper and pencil exercise, is easily applied through use of widely available computers. For example, one of us (M.B.) has developed a system of morphometric analysis using commercially available software (FileMaker Pro, for the Macintosh) that requires only entry of the basic measurement data to print out standard scores that can then be used for correlation with the morphology and the gestational history and to assess normality.

Portions of the Michigan organ weight data were used in previous studies: from 1973-1980 and $50-1,000 \mathrm{~g}$ in Burdi et al. ('81); and 1973-1987 and $400-1,500 \mathrm{~g}$ in Shepard et al. ('88). As shown by Shepard et al. ('88), there were no large differences in the mean organ weights among several studies (Fujikura and Froelich, '72; Tanimura et al., '71; Golbus and Berry, '77; Burdi et al., '81). Comparison of the present data to these same prior studies again shows no major differences in mean organ weights, but the $95 \%$ prediction intervals are considerably different for a number of viscera. When our data are compared with published standards derived from larger/older fetuses and neonates, there are seen to be major differences in a few of the means and most of the prediction intervals. We believe our data, collected systematically under a specific protocol, are the more accurate. It should be noted that these standards apply to measurements made with care and precision; the computer buff's aphorism, "GIGO" (garbage in, garbage out), applies here. It is our experience and opinion, that diligence in the collection of morphometric data requires little in terms of effort and time from the prosector and pays considerable dividends.

\section{LITERATURE CITED}

Burdi, A.R., M. Barr, and W.J. Babler (1981) Organ weight patterns in human fetal development. Hum. Biol., 53:355-66.

Fujikura, T., and L.A. Froelich (1972) Organ-weight brain-weight ratios as a parameter of prenatal growth: A balanced growth theory of visceras. Am. J. Obstet. Gynecol., 112:896-902.

Golbus, M.S., and L.C. Berry (1977) Human fetal development between 90 and 170 days postmenses. Teratology, 15:103-8.

Gruenwald, P., and H.N. Minh (1960) Evaluation of body and organ weights in perinatal pathology: I. Normal standards derived from autopsies. Am. J. Clin. Pathol., 34:247-53.

Hall, J.G., U.G. Froster-Iskenius, and J.E. Allanson (1989) Handbook of Normal Physical Measurements. Oxford Medical Publications, New York.

Johnson, M.P., M. Barr, F. Quershi, A. Drugan, and M.I. Evans (1989) Symmetrical intrauterine growth retardation is not symmetrical: Organ-specific gravimetric deficits in midtrimester and neonatal trisomy 18. Fetal Therapy, 4:110-119.

Larroche, J-C. (1977) Developmental Pathology of the Neonate. Excerpta Medica, Amsterdam, pp. 1-21

Potter, E.L., and J.M. Craig (1975) Pathology of the Fetus and the Infant, 3rd ed. Year Book Medical Publishers, Chicago, pp. 15-24.

Saul, R.A., R.E. Stevenson, R.C. Rogers, S.A. Skinner, L.A. Prouty, and D.B. Flannery (1988) Growth References From Conception to Adulthood. Proceedings of the Greenwood Genetic Center, Greenwood, S.C. (Suppl 1).

Schulz, D.M., D.A. Giordano, and D.H. Schulz (1962) Weights of organs of fetuses and infants. Arch. Pathol., 74:244-50.

Shepard, T.H., M. Shi, G.W. Fellingham, M. Fujinaga, J.M. Fitzsimmons, A.G. Fantel, and M. Barr (1988) Organ weight standards for human fetuses. Pediatr. Pathol., 8:513-524.

Singer, D.B., C.J. Sung, and J.S. Wigglesworth (1991) Fetal growth and maturation: With standards for body and organ development. In: Textbook of Fetal and Perinatal Pathology, Vol. 1. J.S. Wigglesworth and D.B. Singer, eds. Blackwell Scientific Publications, Boston, pp. 11-47.

Tanimura, T., T. Nelson, R.R. Hollingsworth, and T.H. Shepard (1971) Weight standards for organs from early human fetuses. Anat. Rec., 171:227-36.

Zeger, S.L., and S.D. Harlow (1991) Mathematical models from laws of growth to tools for biologic analysis: Fifty years of Growth. Growth, 20:1-21. 